\title{
Preventing diabetic renal disease: the potential reno-protective effects of SGLT2 inhibitors
}

\author{
GIUSEPPE MALTESE, AHMAD ABOU-SALEH, LUIGI GNUDI, JANAKA KARALLIEDDE
}

\begin{abstract}
Diabetic renal disease is associated with increased cardiovascular risk and is one of the leading causes of end-stage renal disease worldwide. A combination of hyperglycaemia and hypertension drives the development and progression of diabetic renal disease, with glomerular hyperfiltration being an early manifestation of the disease process. Sodiumglucose linked transporter 2 (SGLT2) inhibitors represent a novel class of drugs that lower plasma glucose levels through the inhibition of renal proximal tubular glucose uptake and secondary glycosuria.

Clinical evidence that SGLT2 inhibitors attenuate glomerular hyperfiltration is complemented by animal data suggesting that these agents can prevent progression of diabetic renal disease. In clinical studies involving patients with type 1 and type 2 diabetes, SGLT2 inhibition reduces glomerular hyperfiltration and appears (albeit in post-hoc and pooled analyses) to reduce urinary albumin excretion. The longer term potential reno-protective effects of this class of drugs are currently under evaluation in large randomised clinical trials.
\end{abstract}

Br J Diabetes Vasc Dis 2015;15:114-118

Key words: tubuloglomerular feedback, hyperfiltration, diabetic kidney disease, albuminuria, SGLT2 inhibitors

\section{Introduction}

Diabetic renal disease is the leading cause of end stage renal disease (ESRD) in the western world. The number of patients with diabetes mellitus and renal disease remains high and represents an important and challenging public health burden, despite remarkable progress made in reducing the development and progression of diabetic renal disease in recent decades. Indeed, diabetic renal disease occurs in approximately $30-35 \%$ of patients with type 1 or type 2 diabetes. ${ }^{1}$ All-cause mortality in patients

Cardiovascular Division, King's College London, London, UK

Address for correspondence: $\mathrm{Dr}$ Giuseppe Maltese Cardiovascular Division, Franklin Wilkins Building, King's College London, Stamford Street, London SE1 9NH, UK

Tel: +44 (0)2078484464

E-mail: giuseppe.maltese@kcl.ac.uk

http://dx.doi.org/10.15277/bjdvd.2015.030 with diabetic renal disease is nearly 20-40 times higher than that in patients without nephropathy, ${ }^{1,2}$ and renal disease is an independent and powerful risk factor for cardiovascular disease.

Microalbuminuria is the earliest clinical manifestation of diabetic renal injury and serves as a biomarker of renal and vascular injury in diabetes. ${ }^{3}$ Although the terms, "normoalbuminuria", "microalbuminuria" and "macroalbuminuria" (or "clinical albuminuria"), describe different categories of renal injury, it is important to remember that they are part of a continuum within the relationship between albumin excretion and cardiorenal risk. ${ }^{3}$

The evolution of diabetic renal disease proceeds through several distinct but interconnected phases:

- an early phase of physiological abnormalities in renal function

- a microalbuminuria phase

- a clinical phase of persistent clinical albuminuria

- a decline in glomerular filtration rate (GFR) and ultimately ESRD.

SGLT2 inhibitors are a novel class of antidiabetic agents that can potentially reduce blood pressure and weight and also may have other reno-protective effects (Figure 1). The efficacy of SGLT2 inhibitors has been reviewed in detail. ${ }^{4}$ Recent metaanalyses of trials, in which SGLT2 inhibitors were used as monotherapy or add-on treatment, demonstrated a reduction in $\mathrm{HbA}_{1 \mathrm{c}}$ of $0.6-0.7 \%$, together with weight loss of $1.0-3.0 \mathrm{~kg}$ and a small drop in systolic/diastolic blood pressures of $4 \mathrm{mmHg}$ and $2 \mathrm{mmHg}$ respectively. 5,6

The inhibition of glucose reabsorption by SGLT2 inhibitors in the proximal tubule is paralleled by the inhibition of sodium uptake, which is believed to underlie the key beneficial effect of this class of drugs on renal haemodynamics and functional parameters. This review aims to summarise the potential renoprotective effects of SGLT2 inhibitors.

\section{Glomerular hyperfiltration in diabetes}

Glomerular hyperfiltration is defined by a GFR of $125-140 \mathrm{~mL}$ $\min / 1.73 \mathrm{~m}^{2}$, or $>2$ standard deviations above the mean GFR in normal, healthy individuals. ${ }^{7}$ In patients with type 1 diabetes, glomerular hyperfiltration is a risk factor for the development of progressive diabetic kidney disease: the odds of progression to at least microalbuminuria in patients with hyperfiltration were 2.7 fold higher relative to patients with normofiltration. ${ }^{8}$ It can be observed in $25-70 \%$ of patients with type 1 diabetes, often early in the course of diabetes, and it is dependent on associated glycaemic control, age, degree of albuminuria, diabetes duration, younger age of diabetes onset, and pubertal status. ${ }^{7-9}$ The 
Figure 1. Putative mechanisms of renoprotection with SGLT2 inhibitors

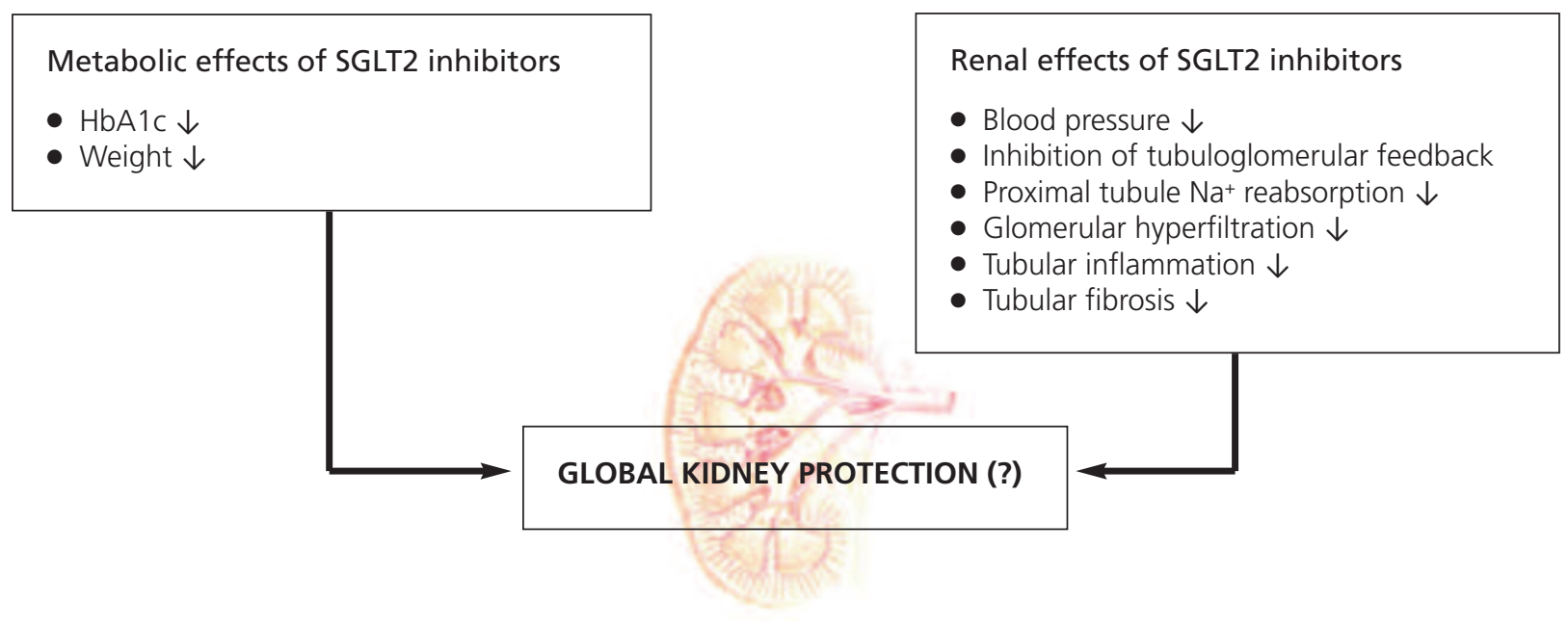

prevalence in patients with type 2 diabetes is lower (5-40\%).7-9 Interestingly, hyperfiltration is also associated with pre-diabetic states such as impaired fasting glycaemia. ${ }^{9}$ The pathogenesis of glomerular hyperfiltration is not fully clear, but haemodynamic and tubular mechanisms are the likely main explanations (Boxes 1 and 2).

Box 1. The haemodynamic hypothesis

Hyperfiltration is the result of changes in afferent (pre-glomerular) and efferent (post-glomerular) arteriolar tone. Hyperglycaemia increases a range of vasoactive mediators and activates pathways involving the renin-angiotensin system, the nitric oxide system, cyclooxygenase 2-derived prostanoids, protein kinase $C$ and endothelin, which result in afferent arteriolar vasodilatation and efferent arteriolar vasoconstriction. ${ }^{7-9}$

Box 2. The tubular hypothesis

Hyperfiltration is initiated by increased sodium reabsorption coupled to glucose transport mediated by SGLT1 and SGLT2 (Figures 2, 3a). ${ }^{9}$ Increased proximal reabsorption of sodium reduces delivery of sodium to the macula densa, which senses this as a decline in effective circulating volume and renal perfusion. This results in a reduction in adenosine generation in the juxtaglomerular apparatus, leading to afferent arteriolar vasodilatation, increased renal perfusion and increased GFR which is termed tubuloglomerular feedback (Figure 2). In diabetes, the reduction in sodium delivery to the distal nephron is a consequence of increased proximal reabsorption of sodium by SGLT2, independent of volume status.

Hyperfiltration has been demonstrated to predict glomerular morphological changes of diabetic renal disease and may contribute to GFR decline. ${ }^{7}$ However, prospective studies and randomised controlled trials are needed to demonstrate that correction of hyperfiltration leads to improved renal outcomes independently of other variables.
Figure 2. Glomerular hyperfiltration mediated by tubuloglomerular feedback

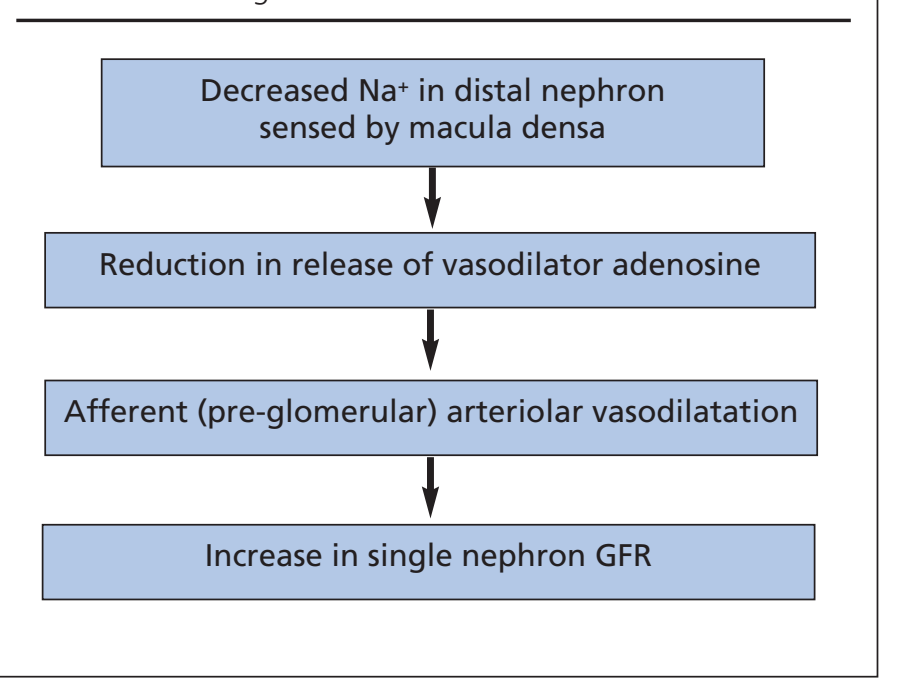

\section{Renal effects of SGLT2 inhibition}

Kidneys of healthy subjects filter about $180 \mathrm{~g}$ of glucose every day and more than $99 \%$ of this glucose is reabsorbed. About $90 \%$ of glucose reabsorption occurs via SGLT2, expressed in the apical epithelium of proximal tubular cells; SGLT1 is mainly expressed in the brush borders of intestinal epithelial cells and in the proximal renal tubule where it contributes to approximately $10 \%$ of glucose reabsorption.

The expression and activity of SGLT2 are up-regulated in both type 1 and type 2 diabetes, contributing to the pathogenesis of hyperglycaemia. ${ }^{15}$ SGLT2 inhibition reduces sodium transport in the proximal tubule and increases sodium delivery to the juxtaglomerular apparatus, which leads to a reduction in glomerular pressure and glomerular hyperfiltration (Figure 3b). ${ }^{16}$ This amelioration in glomerular hyperfiltration is hypothesised to lead to renoprotection. 
Figure 3. Arteriolar tone, $\mathrm{Na}^{+} /$glucose reabsorption and tubuloglomerular feedback (TGF) in early diabetic renal disease (a) and effects of SGLT2 inhibition (b)

a) Hyperfiltration in early diabetic renal disease

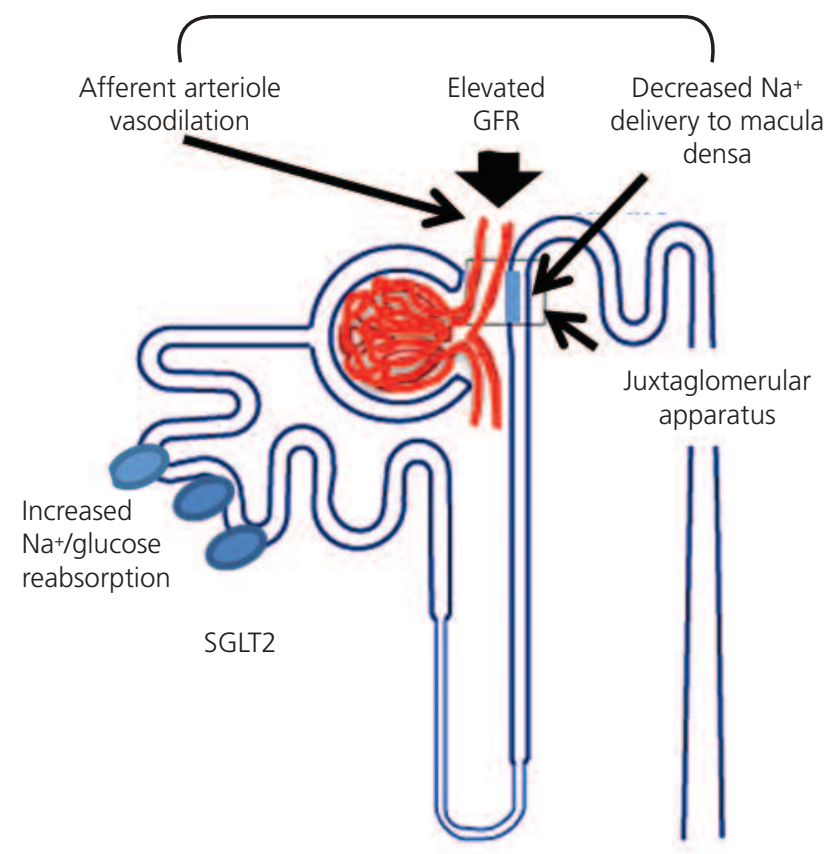

b) SGLT2 inhibition reduces hyperfiltration

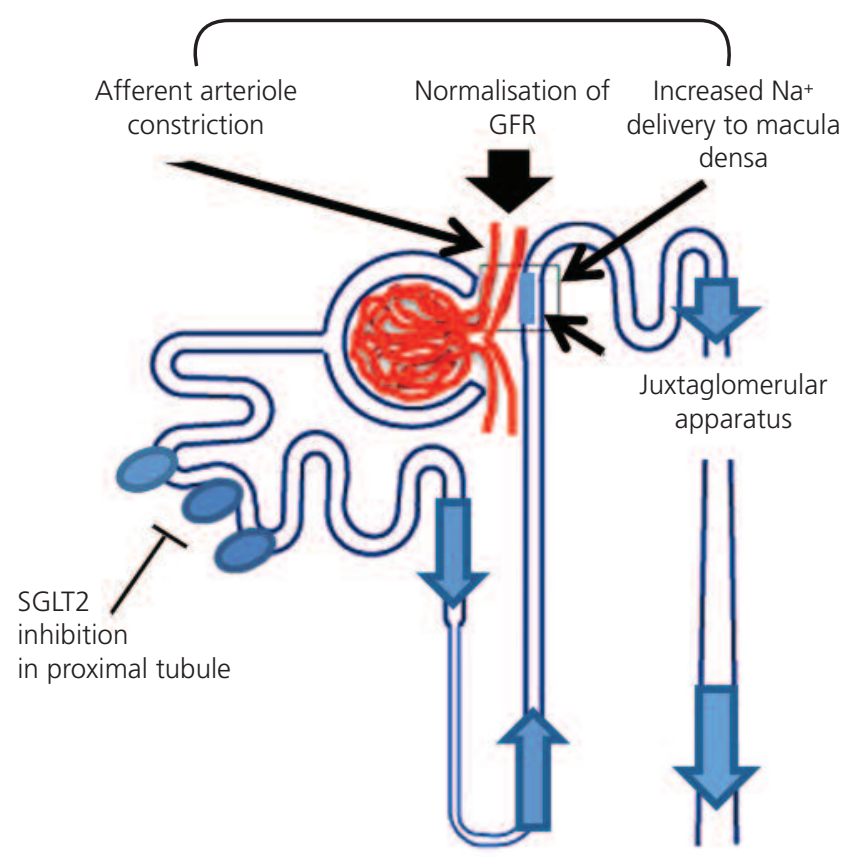

glycosuria
SGLT2 inhibition decreases hyperfiltration and histological evidence of diabetic nephropathy in animal models of type 1 and type 2 diabetes. 9,17 Treatment with a SGLT2 inhibitor in an in vivo model of type 1 diabetes leads to the amelioration of hyperglycaemia-associated hyperfiltration, along with reduced levels of albuminuria, kidney hypertrophy and inflammatory markers. ${ }^{17}$ Interestingly, experimental knockout of SGLT2 in diabetic mice has no effects on the rise of markers of tubular damage such as NGAL and KIM1. ${ }^{18}$ The effects of SGLT2 inhibitors on markers of kidney injury in patients with type 2 diabetes are as yet unknown.

Animal data on SGLT2 blockade and reno-protection have been supported by several recent clinical studies. Inhibition of SGLT2 with empagliflozin (25 mg for 8 weeks) reduced glomerular hyperfiltration in an elegant study conducted in patients with type 1 diabetes, with or without hyperfiltration (GFR >135 mL/ min or 90-134 mL/min, respectively). ${ }^{16}$ GFR dropped significantly in this study only in patients with hyperfiltration, by $33 \mathrm{~mL} / \mathrm{min} /$ $1.73 \mathrm{~m}^{2}$ (during clamped euglycaemia) and $44 \mathrm{~mL} / \mathrm{min} / 1.73 \mathrm{~m}^{2}$ (during hyperglycaemia). Changes in GFR were accompanied by a significant decline in effective renal plasma and renal blood flow and by a significant rise in renal vascular resistance. These findings suggest that the tubuloglomerular feedback plays an important role in glomerular hyperfiltration and SGLT2 inhibitors can attenuate this phenomenon. However, whether these shortterm changes in renal hyperfiltration translate into long-term renal protection is unknown.
In four of the largest trials to date in patients with type 2 diabetes, a mild reduction in eGFR of 1.1-4.8 $\mathrm{mL} / \mathrm{min}$ was observed over 24-104 weeks of follow-up. ${ }^{19-21}$ A greater reduction in urine albumin to creatinine ratio (UACR) was observed with canagliflozin $100 \mathrm{mg}$ versus glimepiride in patients with preserved renal function (eGFR $>55 \mathrm{~mL} / \mathrm{min}$ ), despite equivalent glucose lowering. ${ }^{21}$ However there were greater reductions in blood pressure and body weight with SGLT2 inhibition and hence these data do not provide a clear demonstration of a glucose-independent effect on albuminuria.

In a recent 2-year study, 252 patients with poorly controlled type 2 diabetes and impaired renal function (mean eGFR $45.6 \mathrm{~mL} / \mathrm{min}$ ) were randomised to receive dapagliflozin $5 \mathrm{mg}$ or $10 \mathrm{mg}$, or placebo. Mean eGFR decreased after one week of dapagliflozin, but then remained stable throughout the remainder of the study, with similar mean changes from baseline in eGFR at week 104 for the three groups $(-1.71,-3.50$ and $-2.38 \mathrm{~mL} / \mathrm{min}$, respectively).22 The authors attributed the early drop in eGFR with dapagliflozin as a consequence of a modest anti-diuretic effect and increased tubuloglomerular feedback, with resultant afferent arteriolar vasoconstriction. Whether these early changes in GFR translate to longer term reno-protection remains to be proven. In this study, patients receiving dapagliflozin did not significantly improve glycaemic control but did have lower weight and blood pressure and were more likely to regress to a lower albumin excretion category than patients randomised to placebo. ${ }^{22}$ 


\section{Key messages}

- SGLT2 inhibitors are a new class of oral anti-diabetic drugs with a glycosuric effect that improves glycaemic control, aids weight loss and is associated with reduced blood pressure

- Effects on glomerular hyperfiltration may ameliorate the decline in renal function in diabetes

- The results of ongoing long-term randomised controlled trials are awaited to confirm the renal and cardiovascular effects of SGLT2 inhibitors

Canagliflozin $100 \mathrm{mg}$ and $300 \mathrm{mg}$ were associated with changes from baseline in eGFR $\left(-3.6\right.$ and $-3.9 \mathrm{~mL} / \mathrm{min} / 1.73 \mathrm{~m}^{2}$, respectively) in a 6-month study in 269 patients with type 2 diabetes and eGFR $30-50 \mathrm{~mL} / \mathrm{min} / 1.73 \mathrm{~m}^{2} .{ }^{23} \mathrm{~A}$ modest decrement in eGFR $\left(-1.4 \mathrm{~mL} / \mathrm{min} / 1.73 \mathrm{~m}^{2}\right)$ was noted in those randomised to placebo. Canagliflozin 100 and $300 \mathrm{mg}$ were also associated with significant decreases in UACR compared with placebo in this study (median percent reductions of $-29.9 \%$, $-20.9 \%$, and $-7.5 \%$, respectively). Progression of albuminuria category (from normoalbuminuria to microalbuminuria or macroalbuminuria, or from microalbuminuria to macroalbuminuria) occurred in $5.1 \%$ (canagliflozin $100 \mathrm{mg}$ ), 8.3\% (canagliflozin $300 \mathrm{mg}$ ), and 11.8\% (placebo).

A randomised placebo-controlled double blind trial involving 75 patients with type 2 diabetes showed that reductions in 24-hour systolic blood pressure occurred with dapagliflozin $10 \mathrm{mg} /$ day $(-3.3 \mathrm{mmHg})$ or hydrochlorothiazide $25 \mathrm{mg} /$ day $(-6.6 \mathrm{mmHg})$, compared with $-0.9 \mathrm{mmHg}$ for placebo. ${ }^{24}$ The reduction in blood pressure observed with dapagliflozin was related to a possible diuretic effect secondary to increased sodium excretion. Further information on the efficacy of SGLT2 inhibitors in patients with renal impairment will come from the ongoing clinical trial, "Evaluation of the Effects of Canagliflozin on Renal and Cardiovascular Outcomes in Participants With Diabetic Nephropathy (CREDENCE)". This randomised, double blind placebo-controlled clinical trial (NCT02065791) will investigate the effects of canagliflozin $100 \mathrm{mg} /$ day on the progression of renal impairment and on clinical outcomes (cardiovascular death) in patients with type 2 diabetes, stage 2 or 3 chronic kidney disease and macroalbuminuria, who are receiving the current standard of care, including a RAS blocker.

In summary, SGLT2 inhibitors are a promising class of oral antidiabetic agents. At this stage, it is premature to say if early renal haemodynamic changes induced by these drugs will translate into reduced albumin excretion and reno-protection over the long term. Certainly, the improvement in glycaemic and blood pressure control mediated by SGLT2 inhibition is likely to have a positive impact on preventing and/or delaying the progression of diabetic kidney disease.

\section{Conclusions}

SGLT2 inhibitors are a new class of antidiabetic agents with several mechanisms of action independent of glucose lowering that may offer potential reno-protection in diabetes. However, the results of long-term clinical trials are required before this class of drugs can be considered as an additional therapeutic option in clinical practice for preventing or delaying the progression of diabetic renal disease.

Conflict of interest JK and LG have previously received research grant funding and speaker honoraria from the manufacturers of SGLT2 inhibitors.

Funding No funding was received for this work.

\section{References}

1. Karalliedde J and Viberti GC. Diabetic Nephropathy. 2nd edition. Davies M AS, editor. Oxford: Oxford University Press 2011.

2. Karalliedde $J$ and Viberti GC. Proteinuria in diabetes: bystander or pathway to cardiorenal disease? JASN 2010;21:2020-7. http://dx.doi.org/10.1681/ASN.2010030250

3. Viberti GC, Hill RD, Jarrett RJ et al. Microalbuminuria as a predictor of clinical nephropathy in insulin-dependent diabetes mellitus. Lancet 1982;1:1430-2. http://dx.doi.org/10.1016/S0140-6736(82)92450-3

4. Rosenwasser RF, Sultan S, Sutton D et al. SGLT-2 inhibitors and their potential in the treatment of diabetes. Diabetes Metab Syndr Obes 2013; 6:453-67. http://dx.doi.org/10.2147/DMSO.S34416

5. Musso G, Gambino R, Cassader M et al. A novel approach to control hyperglycemia in type 2 diabetes: sodium glucose co-transport (SGLT) inhibitors: systematic review and meta-analysis of randomized trials. Ann Med 2012;44:375-93.

http://dx.doi.org/10.3109/07853890.2011.560181

6. Vasilakou D, Karagiannis T, Athanasiadou E et al. Sodium-glucose cotransporter 2 inhibitors for type 2 diabetes: a systematic review and meta-analysis. Ann Intern Med 2013;159:262-74.

http://dx.doi.org/10.7326/0003-4819-159-4-201308200-00007

7. Jerums G, Premaratne E, Panagiotopoulos S et al. The clinical significance of hyperfiltration in diabetes. Diabetologia 2010;53:2093-104. http://dx.doi.org/10.1007/s00125-010-1794-9

8. Magee GM, Bilous RW, Cardwell CR et al. Is hyperfiltration associated with the future risk of developing diabetic nephropathy? A meta-analysis. Diabetologia 2009;52:691-7 http://dx.doi.org/10.1007/s00125-009-1268-0

9. Sasson AN, Cherney DZ. Renal hyperfiltration related to diabetes mellitus and obesity in human disease. World J Diabetes 2012;3:1-6. http://dx.doi.org/10.4239/wjd.v3.i1.1

10. Melsom T, Mathisen UD, Ingebretsen OC et al. Impaired fasting glucose is associated with renal hyperfiltration in the general population. Diabetes Care 2011;34:1546-51. http://dx.doi.org/10.2337/dc11-0235

11. ter Maaten JC, Bakker SJ, Serne EH et al. Insulin's acute effects on glomerular filtration rate correlate with insulin sensitivity whereas insulin's acute effects on proximal tubular sodium reabsorption correlate with salt sensitivity in normal subjects. Nepron Dial Transplant 1999;14:2357-63. http://dx.doi.org/10.1093/ndt/14.10.2357

12. Oterdoom LH, de Vries AP, Gansevoort RT et al. Fasting insulin modifies the relation between age and renal function. Nepron Dial Transplant 2007;22:1587-92. http://dx.doi.org/10.1093/ndt/gfm037

13. Wright EM. Renal $\mathrm{Na}(+)$-glucose cotransporters. Am J Physiol Renal Physiol 2001;280:F10-8.

14. Vallon V, Platt KA, Cunard R et al. SGLT2 mediates glucose reabsorption in the early proximal tubule. J Am Soc Nephrol 2011;22:104-12. http://dx.doi.org/10.1681/ASN.2010030246

15. Mather A, Pollock C. Renal glucose transporters: novel targets for hyperglycemia management. Nature Rev Nephrol 2010;6:307-11. http://dx.doi.org/10.1038/nrneph.2010.38

16. Cherney DZ, Perkins BA, Soleymanlou N et al. Renal hemodynamic effect of sodium-glucose cotransporter 2 inhibition in patients with type 1 diabetes mellitus. Circulation 2014;129:587-97. http://dx.doi.org/10.1161/CIRCULATIONAHA.113.005081

17. Vallon V, Gerasimova M, Rose MA et al. SGLT2 inhibitor empagliflozin 
reduces renal growth and albuminuria in proportion to hyperglycemia and prevents glomerular hyperfiltration in diabetic Akita mice. Am J Physiol Renal Physiol 2014;306:F194-204.

http://dx.doi.org/10.1152/ajprenal.00520.2013

18. Ly JP, Onay T, Sison K et al. The Sweet Pee model for Sglt2 mutation. J Am Soc Nephrol 2011;22:113-23. http://dx.doi.org/10.1681/ASN.2010080888

19. List JF, Woo V, Morales E et al. Sodium-glucose cotransport inhibition with dapagliflozin in type 2 diabetes. Diabetes Care 2009;32:650-7. http://dx.doi.org/10.2337/dc08-1863

20. Wilding JP, Woo V, Soler NG et al. Long-term efficacy of dapagliflozin in patients with type 2 diabetes mellitus receiving high doses of insulin: a randomized trial. Ann Intern Med 2012;156:405-15.

http://dx.doi.org/10.7326/0003-4819-156-6-201203200-00003

21. Cefalu WT, Leiter LA, Yoon KH et al. Efficacy and safety of canagliflozin versus glimepiride in patients with type 2 diabetes inadequately controlled with metformin (CANTATA-SU): 52 week results from a randomised, double-blind, phase 3 non-inferiority trial. Lancet 2013;382:941-50. http://dx.doi.org/10.1016/S0140-6736(13)60683-2

22. Kohan DE, Fioretto $P$, Tang $W$ et al. Long-term study of patients with type 2 diabetes and moderate renal impairment shows that dapagliflozin reduces weight and blood pressure but does not improve glycemic control. Kidney Int 2014;85:962-71. http://dx.doi.org/10.1038/ki.2013.356

23. Yale JF, Bakris G, Cariou B et al. Efficacy and safety of canagliflozin in subjects with type 2 diabetes and chronic kidney disease. Diabetes Obes Metab 2013;15:463-73. http://dx.doi.org/10.1111/dom.12090

24. Lambers Heerspink HJ, de Zeeuw D, Wie L et al. Dapagliflozin a glucoseregulating drug with diuretic properties in subjects with type 2 diabetes. Diabetes Obes Metab 2013;15:853-62 http://dx.doi.org/10.1111/dom.12127

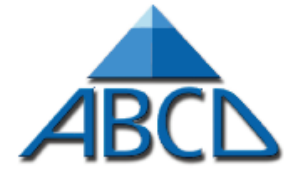

Association of British Clinical Diabetologists

\section{Dapagliflozin (Forxiga) Nationwide Audit in progress}

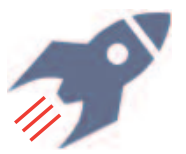

$A B C D$ launched a nationwide audit of dapagliflozin in the UK.

This audit is particularly important with dapaglifozin being the first of a new class of drugs for diabetes, the SGLT2 inhibitors. We have a chance to assess real clinical efficacy and safety of this new type of treatment by pooling our experience nationwide

\section{Does your centre use dapagliflozin (Forxiga)?}

\section{If yes, REGISTER YOUR CENTRE! by contacting abcd.audits@diabetologists.org.uk}

- you are able to analyse your local data easily

- you are invited to enter your patients' data into the online tool on N3 (the NHS secure network)

- the data will be automatically added to the national data in anonymised form

- we can provide easy-to-complete paper proformas for use in clinic if preferred

Please remember: - the more data, the more complete our understanding of this new treatment will be - all contributors will be listed in publications arising from data submission 